\title{
Comparison of Microwave Ablation and Transarterial Chemoembolization for Single-Nodule Hepatocellular Carcinoma Smaller Than 5cm: A Propensity Score Matching Analysis
}

Cancer Management and Research

\author{
Rui Cui (iD) 1,2 \\ Xiao-hui Wang ${ }^{3}$ \\ Chao $\mathrm{Ma}^{4}$ \\ Tong Liu ${ }^{5}$ \\ Zhi-gang Cheng (D) \\ Zhi-yu Han' \\ Fang-yi Liu' \\ Xiao-ling Yu' \\ Jie $\mathrm{Yu}^{\mathrm{l}}, *$ \\ Ping Liang ${ }^{1} *$ \\ 'Department of Interventional \\ Ultrasound, Chinese PLA General \\ Hospital, Beijing 100853, People's \\ Republic of China; ${ }^{2}$ Department of \\ Ultrasonography, The Sixth Affiliated \\ Hospital, Sun Yat-sen University, \\ Guangzhou 510000, People's Republic of \\ China; ${ }^{3}$ Department of Ultrasonography, \\ The First Affiliated Hospital of \\ Zhengzhou University, Zhengzhou \\ 450000, People's Republic of China; \\ ${ }^{4}$ Department of Ultrasonography, \\ Qiqihaer Hospital of Chinese Medicine, \\ Qiqihaer 161000, People's Republic of \\ China; ${ }^{5}$ Department of Ultrasonography, \\ Hebei Chest Hospital, Shijiazhuang \\ 050000, People's Republic of China \\ *These authors contributed equally to \\ this work
}

Correspondence: Ping Liang; Jie Yu Department of Interventional Ultrasound, Chinese PLA General Hospital, 28 Fuxing Road, Beijing 100853, People's Republic of China

Tel +86-10-66939530

Fax +86- $\mid 0-68161218$

Email liangping30I@hotmail.com; yu-jie30I@hotmail.com
Purpose: To compare overall survival (OS) and progression-free survival (PFS) between microwave ablation (MWA) and transarterial chemoembolization (TACE) for solitary hepatocellular carcinoma (HCC) smaller than $5 \mathrm{~cm}$.

Methods: Patients with solitary HCC smaller than $5 \mathrm{~cm}$ who initially underwent MWA or TACE were identified in Chinese PLA General Hospital from June 2010 to October 2015. Propensity score matching (PSM) was performed with a 1:1 matching protocol. OS and PFS were compared by using the log-rank test. After matching, subgroup analysis based on tumor size $(\leq 3 \mathrm{~cm} / 3.1-5 \mathrm{~cm})$ was also conducted. Prognostic factors for OS and PFS were assessed with Cox proportional hazard regression model.

Results: A total of 202 patients (MWA, n=120; TACE, n=82) were identified. After matching, 116 patients were included (58 patients for each treatment group). MWA provided significantly better OS and PFS than TACE for both the entire cohort (OS, $\mathrm{P}<0.001$; PFS, $\mathrm{P}<0.001)$ and the matched cohort (OS, $\mathrm{P}=0.015$; PFS, $\mathrm{P}<0.001)$. Subgroup analysis showed that among patients with tumor of $3 \mathrm{~cm}$ or less, the MWA group had significantly better OS $(\mathrm{P}=0.027)$ and PFS $(\mathrm{P}=0.008)$ than the TACE group. Multivariate Cox regression analysis showed TACE was associated with worse OS (hazard ratio, 2.385; 95\% CI: 1.427, 3.985; $\mathrm{P}=0.001$ ) and PFS (hazard ratio, 2.567; 95\% CI: 1.820, 3.622; $\mathrm{P}<0.001$ ).

Conclusion: MWA outperformed TACE for solitary HCC smaller than $5 \mathrm{~cm}$ in OS and PFS. For single tumors less than $5 \mathrm{~cm}$, especially those smaller ones $(\leq 3 \mathrm{~cm})$, priority should be given to MWA when making treatment options between MWA and TACE.

Keywords: hepatocellular carcinoma, transarterial chemoembolization, microwave ablation, overall survival, progression-free survival

\section{Precis' Statement}

This paper reported the comparative results in therapeutic effectiveness between MWA and TACE for solitary HCC smaller than $5 \mathrm{~cm}$.

\section{Introduction}

Hepatocellular carcinoma (HCC) is the fifth most common cancer and the second most frequent cause of cancer mortality globally. ${ }^{1}$ As suggested by clinical practice guidelines of American Association for the Study of Liver Diseases (AASLD), ${ }^{2}$ European Association for the Study of the Liver (EASL) ${ }^{3}$ and 
National comprehensive Cancer Network (NCCN), ${ }^{4}$ thermal ablation dominated by radiofrequency ablation (RFA) is an alternative option for patients with early HCC, especially for those ineligible resection or liver transplantation. Microwave ablation (MWA), as a relatively new ablation technique, showed promising results due to its satisfactory treatment controllability and overall coagulation performance. ${ }^{5,6}$ Therefore, in latest 10 years, MWA has found an increasingly application for early stage $\mathrm{HCC}^{7}$ and gradually becoming popular in Europe and the USA. ${ }^{8}$

Transarterial chemoembolization (TACE), despite of being the first-line therapy for intermediate-stage HCC, is also used in patients with early stage HCC as a bridge to liver transplantation (LT) or a treatment option when LT, hepatic resection (HR), and ablation are not possible, in keeping with the stage migration strategy. ${ }^{9}$ As mentioned in EASL guidelines, in daily clinical practice, TACE is widely applied outside intermediate HCCs. ${ }^{3,10}$ Approximately, $22-43 \%$ of TACEs are performed in early stage ${ }^{3}$ and some studies reported that similar to RFA or MWA, repeat TACE can realize a complete ablation for early stage HCC. ${ }^{11,12}$ In treating early stage $\mathrm{HCC}$, there were six studies comparing thermal ablation and TACE. ${ }^{13-18}$ However, to our best knowledge, there is no research comparing MWA and TACE for early stage HCC and the comparison result in longterm treatment outcome between TACE and thermal ablation is not very clear. Hence, we conducted this study to compare the overall survival (OS) and progression-free survival (PFS) between MWA and TACE for patients with solitary HCC less than $5 \mathrm{~cm}$.

\section{Materials and Methods}

\section{Patients}

This retrospective study was approved by the institutional review board of Chinese PLA General Hospital and complied with the Declaration of Helsinki. Procedure-specific informed consent was obtained from each patient. Patients were selected from HCC patients who underwent TACE or MWA at Chinese PLA General Hospital, from June 2010 to October 2015.

Patient selection was performed with the following criteria: 1) diagnosis of HCC confirmed by liver biopsy or made clinically according to the European Association for the Study of the Liver (EASL) imaging criteria; 2) single nodule with a diameter $<5 \mathrm{~cm}$; 3) patients were initially treated with MWA or TACE; 4) Child-Pugh class A or B; and 5) Eastern Cooperative Oncology Group (ECOG) performance status of 0 to 2. The exclusion criteria included: 1) serious comorbidity including malignant tumors other than HCC; 2) evidence of gross vascular invasion, portal thrombosis or extrahepatic metastases at diagnosis; and 3) underwent resection or liver transplantation after MWA or TACE.

In total, $202 \mathrm{HCC}$ patients meeting these criteria were identified using the protocol shown in Figure 1. Of these, 120 and 82 patients were initially treated with MWA or TACE, respectively. In the 82 patients who underwent TACE, surgery and thermal ablation could not be performed in the following cases: 1) ineligible for surgery due to inadequate hepatic functional conditions (bilirubin level, portal hypertension, and ascites) or extrahepatic comorbidities; 2) increasing risk of thermal injury or inadequate safe margin due to tumor location (close to diaphragm, gallbladder, heart or under the capsule); and 3) refusing HR and MWA due to advanced age, financial concerns or individual preferences after a full explanation of the advices from practice guidelines..$^{2-4}$

\section{Procedures}

MWA was performed by four specialized interventional doctors (P.L. and X.L.Y., each with 20 years of experience; Z.G.C. and Z.Y.H., each with 15 years of experience). After general anesthesia, MWA was performed under standard hemodynamic monitoring using the microwave ablation system (KY-2000; Canyou Medical Instruments, Nanjing, China), which consists of a microwave generator, flexible coaxial cables and cooled shaft antennae. For tumors less than $2.0 \mathrm{~cm}$, one antenna was inserted; for tumors measuring $2.0 \mathrm{~cm}$ or larger, two antennae were inserted with the distance of 1.0 to $2.5 \mathrm{~cm}$. During the MWA procedure, the microwave energy application was set at $40-60 \mathrm{~W}$ for 5-10 mins in a session. Immediately following the MWA procedure, contrast-enhanced ultrasound (CEUS) was conducted to evaluate the completeness of the ablation. MWA was considered to be complete until the hyperechoic ablation zone completely covered the entire tumor in CEUS. An additional session of MWA was performed if the CEUS suggested an incomplete ablation.

TACE was conducted by two specialized interventional radiologists (M.Q.W. with 25 years of experience and F.Y.L. with 15 years of experience). After intravenous moderate sedation and local anesthesia, a 2.6-Fr microcatheter (Progreat, Terumo Corporation) was used to select the tumor feeders at the level of the segmental or 
4777 HCC patients treated by MWA or TACE from June 2010 to October 2015 at Chinese PLA General Hospital

674 patients treated with MWA or TACE for solitary $\mathrm{HCC} \leq 5 \mathrm{~cm}$ assessed for eligibility

Excluded $(\mathrm{n}=472)$

- Evidence of vascular invasion, portal thrombosis or extrahepatic metastases at diagnosis $(n=347)$

- Subsequently underwent HR or LT $(n=119)$

- serious comorbidity other than HCC $(n=6)$

120 patients treated with MWA
82 patients treated with TACE

Figure I Flowchart of patient selection.

subsegmental artery. Then, a mixture of iodized oil (Lipiodol; Laboratoire Andre Guerbet, Aulnay-sous-Bois, France) and doxorubicin hydrochloride (Adriamycin; Pharmacia \& Upjohn, Peapack, NJ, USA) was infused through the feeding vessels. The dosage of doxorubicin hydrochloride and iodized oil depended on tumor size and vascularity. Thereafter, the feeders were embolized with gelatin sponge pledgets (Jinling Pharmaceutical Limited, Nanjing, China). The TACE procedure was ended until complete stagnation of feeding arterial flow was achieved.

Both MWA and TACE were performed during standard hemodynamic monitoring after intravenous anesthesia was achieved. All patients were followed up via combining contrast-enhanced imaging (CT or MR imaging), liver function test and levels of $\alpha$-fetoprotein (AFP) at 1, 3, 6, 9 and 12 months after the procedure and thereafter every 6 months. Treatment response was assessed at 1 month after TACE or MWA based on the modified Response Evaluation Criteria in Solid Tumors (mRECIST), which include complete response (CR), partial response (PR), stable disease (SD) and progressive disease (PD). ${ }^{19}$ If
CR was not achieved after MWA or TACE, patients would accept systematic therapy with sorafenib.

\section{Data Measurement}

Clinical and laboratory data were collected via reviewing the electronic medical records. Child-Pugh class and model for end-stage liver disease (MELD) score ${ }^{20}$ were assessed based on the collected clinical and laboratory variables. The primary outcomes were overall survival (OS) and progression-free survival (PFS), both calculated from date of initial treatment to date of death and date of disease progression, respectively, or to October 31, 2018. In the assessment of PFS, disease progression included the development of local tumor progression, distant hepatic progression (the appearance of one or more new lesions) and extrahepatic progression. For patients evaluated as PD, the PFS was calculated as 1 month; for patients evaluated as $\mathrm{CR}, \mathrm{PR}$ or $\mathrm{SD}$, the PFS was calculated as the method described above. The secondary outcomes included treatment response and complications. Major complication was 
defined as an event that result in substantial morbidity and disability that increases the level of care, or results in hospital admission, or substantially lengthens the hospital stay. ${ }^{21,22}$

\section{Statistical Analysis}

Data were presented as mean \pm SDs for continuous variables and as percentages for categorical variables according to treatment modality grouping. Continuous variables were compared with Kruskal-Wallis test, and categorical variables were compared with chi-square test. OS and PFS were plotted by means of the Kaplan-Meier method and compared with the log-rank test. To balance the baseline clinical variables and control the confounder bias, propensity score matching (PSM) was applied. Propensity score was estimated by generating a logistic regression model with the treatment options as the dependent variable and baseline characteristics including age, sex, tumor size, Child-Pugh class, serum AFP level and the presence of liver cirrhosis as covariates. Matching was performed with a 1:1 matching protocol with the inverse probabilities of treatment (IPTW) and caliper width was set as 0.05 standard deviations. Standardized differences were estimated for the balanced baseline covariates before and after matching to exam balance. Standardized differences of less than $10 \%$ for a given covariate indicate a relatively small imbalance. Before and after PSM, OS, PFS and treatment response were compared between the MWA group and the TACE group. For the matched cohort, subgroup analysis based on tumor size was conducted to compare treatment response, OS and PFS between MWA and TACE. Prognostic factors for OS and PFS were assessed by using Cox proportional hazard models for all patients for the entire cohort before PSM. Data were analyzed with the use of statistical packages R (The R Foundation; http://www.r-project.org; version 3.1.2 2014-10-31) and Empower (R) (www.empowerstatus. com; X\&Y Solutions Inc.).

\section{Results}

\section{Baseline Characteristics of Patients}

The baseline characteristics of patients are summarized in Table 1. Before PSM, patients who underwent TACE had a higher prevalence of ascites $(14.6 \%$ vs $2.5 \%, \mathrm{P}=0.002)$, a higher frequency of Child-Pugh B $(11.0 \%$ vs $0.7 \%$, $\mathrm{P}=0.008)$ and a higher proportion of tumors larger than $3 \mathrm{~cm}(68.3 \%$ vs $29.2 \%, \mathrm{P}<0.001)$ than patients who underwent MWA. After PSM, a total of 58 patients from each group were matched by applying one-to-one matching. Variables of baseline characteristics showed no significant differences between the two groups.

\section{Treatment Response}

In terms of the number of procedures, the sessions in the MWA group were less than those in the TACE group for the unmatched cohort $(1.1 \pm 0.3$ vs $1.2 \pm 0.5, \mathrm{P}=0.019)$ but not for the matched cohort $(1.1 \pm 0.3$ vs $1.3 \pm 0.6$, $\mathrm{P}=0.056)$ (Table S1).

As for the treatment response, before PSM, CR rates of the TACE group were $69.5 \%$, significantly lower than that of the MWA group $(98.3 \%, \mathrm{P}<0.001)$. After PSM, significant differences remained between the two groups (TACE vs MWA, $72.4 \%$ vs $100.0 \%, \mathrm{P}<0.001$ ) (Table S1).

\section{Overall Survival}

For the entire cohort, the median follow-up duration of the MWA and TACE groups was 44.0 months (range, 3.0-100.0 months) and 40.5 months (range, 3.0-89.0 months), respectively $(\mathrm{P}=0.181)$. During the follow-up, 28 of 120 (23.3\%) patients in the MWA group, 44 of $82(53.7 \%)$ in the TACE group died. The 1-, 3-, and 5-year cumulative probabilities of OS were $97.5 \%, 84.3 \%$ and $71.6 \%$ in the MWA group and $85.2 \%, 58.2 \%, 43.3 \%$ in the TACE group, respectively $(\mathrm{P}<0.001)$ (Figure $\mathrm{S} 1)$. After PSM, by the end of follow-up, $16(27.6 \%)$ patients in the MWA group and $32(55.2 \%)$ patients in the TACE group died. A comparison in OS of the two groups is shown in Figure 2. The cumulative OS rate at 1,3 , and 5 years were $96.5 \%, 76.4 \%$, and $70.2 \%$ for the patients in MWA group and $87.8 \%, 59.1 \%$, and $43.6 \%$ for patients in TACE group with significant difference $(\mathrm{P}=0.015)$. In subgroup analysis based on tumor size, MWA provides better OS than TACE for patients with tumor of $3 \mathrm{~cm}$ or smaller $(\mathrm{P}=0.027)$ but not for those with tumor of $3.1-5 \mathrm{~cm}(\mathrm{P}=$ 0.244) (Figure 3).

In multivariate Cox regression analysis, advanced age (hazard ratio, 2.937; 95\% CI: 1.796, 4.803; $\mathrm{P}<0.001$ ), male sex (hazard ratio, 2.235; 95\% CI: 1.173, 4.392; $\mathrm{P}=0.020$ ), tumor of $3.1-5 \mathrm{~cm}$ (hazard ratio, $1.792 ; 95 \%$ CI: 1.075 , 2.987; $\mathrm{P}=0.025$ ), and TACE (hazard ratio, 2.385; 95\% CI: $1.427,3.985 ; \mathrm{P}=0.001)$ were identified as independent predictors of poor survival (Table 2).

\section{Progression-Free Survival}

For the entire cohort, disease progression was diagnosed in 66 of $120(55.0 \%)$ patients in the MWA group and 71 of 
Table I Baseline Characteristics of the Patients Before and After PSM

\begin{tabular}{|c|c|c|c|c|c|c|}
\hline \multirow[t]{2}{*}{ Parameter } & \multicolumn{3}{|c|}{ Unmatched Cohort } & \multicolumn{3}{|c|}{ Matched Cohort } \\
\hline & $\begin{array}{l}\text { MWA Group } \\
(n=120)\end{array}$ & $\begin{array}{l}\text { TACE Group } \\
(n=82)\end{array}$ & P Value & $\begin{array}{l}\text { MWA Group } \\
(n=58)\end{array}$ & $\begin{array}{l}\text { TACE Group } \\
(n=58)\end{array}$ & P Value \\
\hline Age $(\leq 60 y />60 y)$ & $77 / 43$ & $45 / 37$ & 0.185 & $34 / 24$ & $35 / 23$ & 0.850 \\
\hline Sex (female/male) & $19 / 101$ & $16 / 66$ & 0.498 & $9 / 49$ & $9 / 49$ & 1.000 \\
\hline $\begin{array}{l}\text { Tumor size }(\mathrm{cm}) \\
\leq 3 \\
>3\end{array}$ & $\begin{array}{l}85(70.8 \%) \\
35(29.2 \%)\end{array}$ & $\begin{array}{l}26(31.7 \%) \\
56(68.3 \%)\end{array}$ & $<0.001$ & $\begin{array}{l}23(39.7 \%) \\
35(60.3 \%)\end{array}$ & $\begin{array}{l}24(4 \mathrm{I} .4 \%) \\
34(58.6 \%)\end{array}$ & 0.850 \\
\hline HBV $( \pm)$ & $108 / 12$ & $67 / 15$ & 0.089 & $50 / 8$ & $48 / 10$ & 0.798 \\
\hline $\mathrm{HCV}( \pm)$ & $8 / 112$ & $12 / 70$ & 0.091 & $7 / 51$ & $9 / 49$ & 1.000 \\
\hline Cirrhosis (absent/present) & $12 / 108$ & $7 / 75$ & 0.810 & $5 / 53$ & $6 / 52$ & 0.751 \\
\hline Child-Pugh class (A/B) & $118 / 2$ & $73 / 9$ & 0.008 & $56 / 2$ & $55 / 3$ & 1.000 \\
\hline $\begin{array}{l}\alpha \text {-fetoprotein }(\mu \mathrm{g} / \mathrm{L}) \\
\leq 400 \\
>400 \\
\text { Ascites (absent/present) }\end{array}$ & $\begin{array}{l}106(88.3 \%) \\
14(11.7 \%) \\
117 / 3\end{array}$ & $\begin{array}{l}67(81.7 \%) \\
15(18.3 \%) \\
70 / 12\end{array}$ & $\begin{array}{l}0.187 \\
0.002\end{array}$ & $\begin{array}{l}47(81.0 \%) \\
11(19.0 \%) \\
55 / 3\end{array}$ & $\begin{array}{l}47(81.0 \%) \\
11(19.0 \%) \\
54 / 4\end{array}$ & $\begin{array}{l}1.000 \\
1.000\end{array}$ \\
\hline $\begin{array}{l}\text { Esophageal varix (absent/ } \\
\text { present) }\end{array}$ & $94 / 26$ & $60 / 22$ & 0.397 & $42 / 16$ & $44 / 14$ & 0.672 \\
\hline Serum albumin $(\mathrm{g} / \mathrm{dL})$ & $39.6 \pm 4.6$ & $38.8 \pm 5.3$ & 0.348 & $39.4 \pm 4.8$ & $39.5 \pm 4.8$ & 0.949 \\
\hline Total bilirubin (mg/dL) & $1.0 \pm 0.6$ & $\mathrm{I} . \mathrm{I} \pm 0.5$ & 0.057 & $1.0 \pm 0.7$ & $1.0 \pm 0.5$ & 0.150 \\
\hline $\begin{array}{l}\text { International Normalized Ratio } \\
\text { (INR) }\end{array}$ & $\mathrm{I} . \mathrm{I} \pm 0.2$ & $I . I \pm 0.1$ & 0.891 & $1.2 \pm 0.2$ & $1.1 \pm 0.1$ & 0.713 \\
\hline Creatinine (mg/dL) & $0.9 \pm 0.4$ & $0.8 \pm 0.2$ & 0.294 & $0.9 \pm 0.5$ & $0.8 \pm 0.2$ & 0.584 \\
\hline MELD score & $5.7 \pm 4.4$ & $5.4 \pm 2.8$ & 0.997 & $6.2 \pm 5.4$ & $5.5 \pm 3.0$ & 0.989 \\
\hline ECOG performance status $(0 / \geq 1)$ & $88 / 32$ & $59 / 23$ & 0.828 & $44 / 14$ & $47 / 11$ & 0.498 \\
\hline Diabetes (absent/present) & $98 / 22$ & $66 / 16$ & 0.833 & $46 / 12$ & $48 / 10$ & 0.636 \\
\hline Hypertension (absent/present) & $98 / 22$ & $58 / 24$ & 0.069 & $46 / 12$ & $44 / 14$ & 0.656 \\
\hline $\begin{array}{l}\text { Coronary heart disease (absent/ } \\
\text { present) }\end{array}$ & $112 / 8$ & $79 / 3$ & 0.530 & $54 / 4$ & $56 / 2$ & 0.679 \\
\hline
\end{tabular}

$82(86.6 \%)$ in the TACE group. The cumulative PFS rates at 1, 3 and 5 years were significantly better in the MWA group compared with those in the TACE group $(79.7 \%$, $52.9 \%$ and $32.4 \%$ vs $60.5 \%, 15.2 \%$ and $8.5 \%, \mathrm{P}<0.001)$ (Figure S2). For the matched cohort, disease progression occurred in $34(58.6 \%)$ patients in the MWA group and 52 $(89.7 \%)$ in the TACE group during the follow-up. Consistent with the comparison results before PSM, the 1-, 3-, and 5-year cumulative PFS rates of the MWA group were significantly greater than those of the TACE group $(70.0 \%, 47.5 \%$ and $31.5 \%$ vs $58.9 \%, 11.5 \%$ and $4.6 \%$,
$\mathrm{P}<0.001$ ) (Figure 4). In subgroup analysis, the cumulative PFS rates were significantly higher in the MWA group than those in the TACE group for both the tumors of $3 \mathrm{~cm}$ or smaller $(\mathrm{P}=0.008)$ and the tumors of $3.1-5 \mathrm{~cm}$ $(\mathrm{P}=0.009)$ (Figure 5).

As Table 2 shows, in multivariate Cox regression analysis, TACE was an independent risk factors associated with worse PFS (hazard ratio, 2.567; 95\% CI: 1.820, 3.622; $\mathrm{P}<0.001)$ after adjusting possible risk factors including age (hazard ratio, 1.548; 95\% CI: 1.080, 2.220; $\mathrm{P}=0.017$ ), sex (hazard ratio, 1.608; 95\% CI: 0.996, 2.597; 


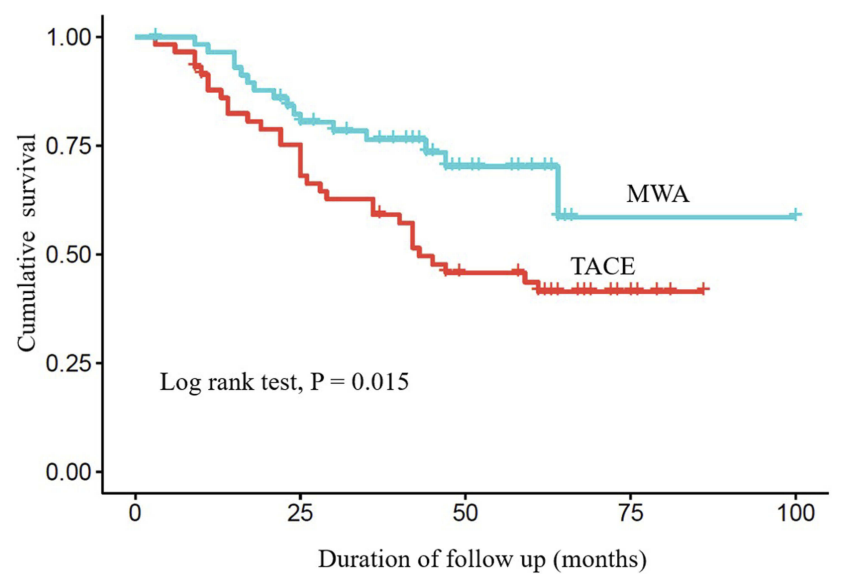

Figure 2 Comparison of the overall survival of HCC patients between the MWA group and the TACE group after PSM. Significantly better survival was found for HCC patients undergoing MWA.

$\mathrm{P}=0.052$ ), HBV infection (hazard ratio, 1.536; 95\% $\mathrm{CI}$ : $0.893,2.643 ; \mathrm{P}=0.121$ ) and the presence of comorbid coronary heart disease (hazard ratio, 0.504 ; 95\% CI: $0.201,1.261 ; \mathrm{P}=0.143$ ).

\section{Major Complications}

There was no significant difference in complication incidence between TACE and MWA (TACE vs MWA, $3.3 \%$ vs $2.4 \%, \mathrm{P}=0.713$ ). Of the 82 patients who underwent TACE, one patient developed liver abscess and one patient developed femoral artery pseudoaneurysm. Of the 120 patients who underwent MWA, four patients developed major complications including hydrothorax $(n=2)$, ascites $(n=1)$ and liver abscess $(n=1)$. Patients with femoral artery pseudoaneurysm were treated with ultrasound-guided thrombin injection; patients with hydrothorax, ascites or liver abscess were treated with ultrasound-guided percutaneous drainage, and conservative treatment was given when necessary. All these complications were relieved before discharge. No lifethreatening complications were observed after either procedure.

\section{Discussion}

MWA, despite of being a recent development in the field of tumor ablation, has been proposed in clinical guidelines $^{2-4}$ as an effective intervention for HCC patients. According to a latest meta-analysis including 9 clinical studies, for HCC in BCLC stage 0 and stage A, MWA is comparable to hepatic resection in terms of OS and PFS, and is associated with shorter operation time, less blood loss amount and fewer complications. ${ }^{23}$ Besides, comprehensive analysis on comparing MWA and RFA for early stage HCC also suggested the similar effectiveness with an apparent superiority of MWA in larger tumors $(3-5 \mathrm{~cm}){ }^{24}$ Therefore, MWA is a promising minimally invasive treatment for early HCC, especially for the larger tumors. Meanwhile, TACE, despite of being recommended for intermediatestage $\mathrm{HCC}$ in the guidelines, has also been a valid option for patient ineligible for curative treatment with early-stage HCC in clinical practice. ${ }^{25,26}$ However, there is a paucity of study comparing therapeutic effectiveness between MWA and TACE for HCC in early-stage. Hence, to present more clinical reference of the treatment allocation for early-stage HCC, we conducted this retrospective study.
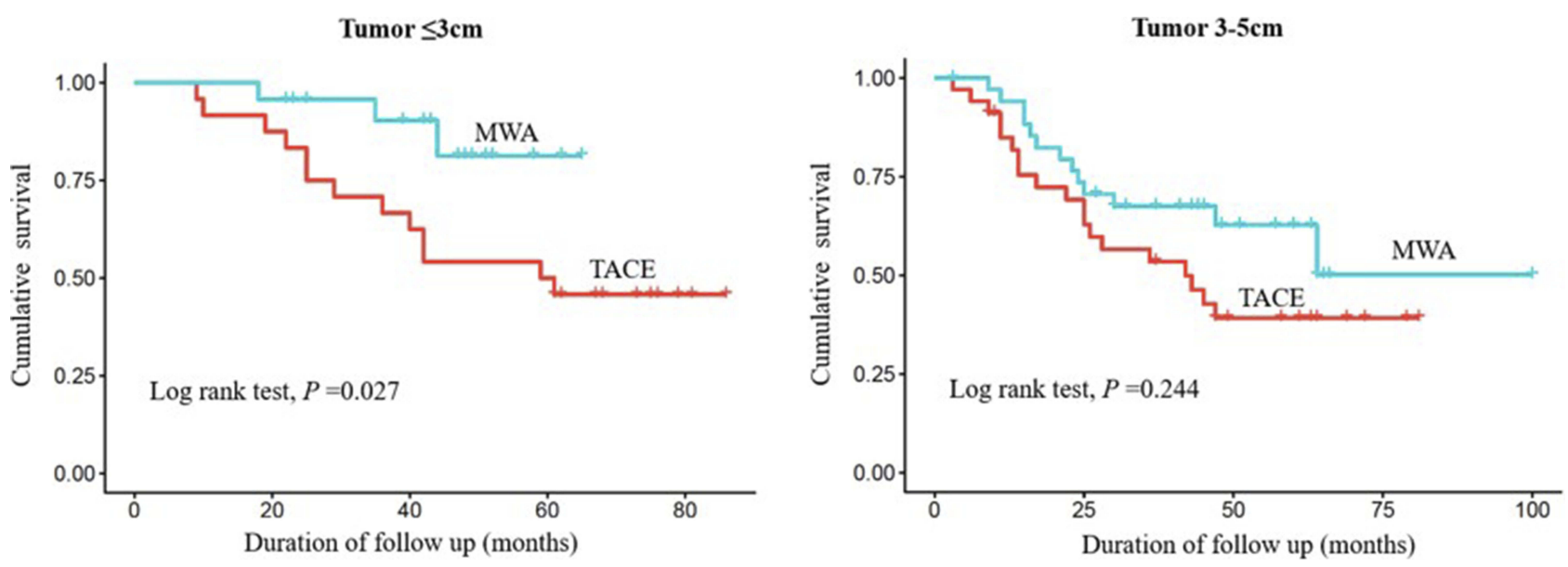

Figure 3 Subgroup analysis of the overall survival of HCC patients undergoing MWA or TACE based on the tumor size ( $\leq 3$ or $3.1-5 \mathrm{~cm})$. MWA provides better OS than TACE for patients with tumor of $3 \mathrm{~cm}$ or smaller but not for those with tumor of $3.1-5 \mathrm{~cm}$. 
Table 2 Univariate and Multivariate Analyses of Prognostic Factors for Overall Survival and Progression-Free Survival

\begin{tabular}{|c|c|c|c|c|c|c|c|c|}
\hline \multirow[t]{3}{*}{ Variables } & \multicolumn{4}{|c|}{ Overall Survival } & \multicolumn{4}{|c|}{ Progression-Free Survival } \\
\hline & \multirow{2}{*}{$\begin{array}{l}\text { Univariate } \\
\text { Analysis } \\
\text { P Value }\end{array}$} & \multicolumn{3}{|c|}{ Multivariate Analysis } & \multirow{2}{*}{$\begin{array}{l}\text { Univariate } \\
\text { Analysis }\end{array}$} & \multicolumn{3}{|c|}{ Multivariate Analysis } \\
\hline & & $\begin{array}{l}\text { Hazard } \\
\text { Ratio }\end{array}$ & $95 \% \mathrm{Cl}$ & P Value & & $\begin{array}{l}\text { Hazard } \\
\text { Ratio }\end{array}$ & $95 \% \mathrm{Cl}$ & P Value \\
\hline Age (>60y vs $\leq 60 y)$ & $<0.001$ & 2.937 & $1.796,4.803$ & $<0.001$ & 0.055 & 1.548 & $1.080,2.220$ & 0.017 \\
\hline Sex (M vs F) & 0.009 & 2.235 & I.137, 4.392 & 0.020 & 0.118 & 1.608 & $0.996,2.597$ & 0.052 \\
\hline $\begin{array}{l}\text { Treatment modality (TACE vs } \\
\text { MWA) }\end{array}$ & 0.004 & 2.385 & I.427, 3.985 & 0.001 & $<0.001$ & 2.567 & $1.820,3.622$ & $<0.001$ \\
\hline HBV (+ vs -) & 0.995 & $\ldots$ & $\ldots$ & $\ldots$ & 0.428 & 1.536 & $0.893,2.643$ & 0.121 \\
\hline $\mathrm{HCV}(+\mathrm{vs}-)$ & 0.996 & $\ldots$ & $\ldots$ & $\ldots$ & 0.945 & $\ldots$ & $\ldots$ & $\ldots$ \\
\hline Serum albumin $(g / L)$ & 0.451 & $\ldots$ & $\ldots$ & $\ldots$ & 0.710 & $\ldots$ & $\ldots$ & $\ldots$ \\
\hline Creatinine (mg/dL) & 0.650 & $\ldots$ & $\ldots$ & $\ldots$ & 0.304 & $\ldots$ & $\ldots$ & $\ldots$ \\
\hline Total bilirubin (mg/dL) & 0.322 & $\ldots$ & $\ldots$ & $\ldots$ & 0.249 & $\ldots$ & $\ldots$ & $\ldots$ \\
\hline $\begin{array}{l}\text { International Normalized Ratio } \\
\text { (INR) }\end{array}$ & 0.503 & $\ldots$ & $\ldots$ & $\ldots$ & 0.999 & $\ldots$ & $\ldots$ & $\ldots$ \\
\hline Cirrhosis (+ vs - ) & 0.476 & $\ldots$ & $\ldots$ & $\ldots$ & 0.566 & $\ldots$ & $\ldots$ & $\ldots$ \\
\hline Ascites (+ vs - ) & 0.373 & $\ldots$ & $\ldots$ & $\ldots$ & 0.695 & $\ldots$ & $\ldots$ & $\ldots$ \\
\hline Esophageal varix (+ vs -) & 0.269 & $\ldots$ & $\ldots$ & $\ldots$ & 0.804 & $\ldots$ & $\ldots$ & $\ldots$ \\
\hline Child-Pugh class (B vs A) & 0.955 & $\ldots$ & $\ldots$ & $\ldots$ & 0.781 & $\ldots$ & $\ldots$ & $\ldots$ \\
\hline MELD score & 0.894 & $\ldots$ & $\ldots$ & $\ldots$ & 0.768 & $\ldots$ & $\ldots$ & $\ldots$ \\
\hline $\begin{array}{l}\text { ECOG performance status (>I } \\
\text { vs } \leq 1 \text { ) }\end{array}$ & 0.915 & $\ldots$ & $\ldots$ & $\ldots$ & 0.595 & $\ldots$ & $\ldots$ & $\ldots$ \\
\hline Hypertension (+ vs -) & 0.226 & 1.496 & $0.891,2.512$ & 0.128 & 0.692 & $\ldots$ & $\ldots$ & $\ldots$ \\
\hline Diabetes (+ vs - ) & 0.493 & $\ldots$ & $\ldots$ & $\ldots$ & 0.222 & $\ldots$ & $\ldots$ & $\ldots$ \\
\hline Coronary heart disease (+ vs - ) & 0.896 & $\ldots$ & $\ldots$ & $\ldots$ & 0.226 & 0.504 & $0.201,1.261$ & 0.143 \\
\hline Tumor Dmax ( $>3 \mathrm{~cm}$ vs $\leq 3 \mathrm{~cm})$ & 0.010 & 1.792 & $1.075,2.987$ & 0.025 & 0.399 & $\ldots$ & $\ldots$ & $\ldots$ \\
\hline $\begin{array}{l}\alpha \text {-Fetoprotein }(>400 \mu g / L \text { vs } \\
\leq 400 \mu g / L)\end{array}$ & 0.123 & 1.576 & $0.879,2.827$ & 0.127 & 0.843 & $\ldots$ & $\cdots$ & $\cdots$ \\
\hline
\end{tabular}

Our results showed that MWA has better survival benefits than TACE in OS for solitary HCC of $5 \mathrm{~cm}$ or less and the superiority of MWA was significant in subgroup analysis of tumors of $3 \mathrm{~cm}$ or less. Similarly, previous study also reported that the other thermal ablation technique, RFA was superior to TACE for patients with smaller tumor burden. ${ }^{16}$ Hence, this finding suggests that HCC patients with smaller solitary tumor may be better candidates for MWA compared with TACE.
In terms of PFS, the results suggested that for singlenodule $\mathrm{HCC}$ of $5 \mathrm{~cm}$ or smaller, TACE was inferior to MWA and was an independent risk factor for worse PFS. This is similar with the results of Lee's study ${ }^{14}$ that TACE was a negative factor for PFS in treating single HCC $(3-5 \mathrm{~cm})$. A possible reason for the better local tumor control of MWA was its better treatment response. In fact, the efficacy of TACE is influenced by many factors including feeding arterials and tumor 


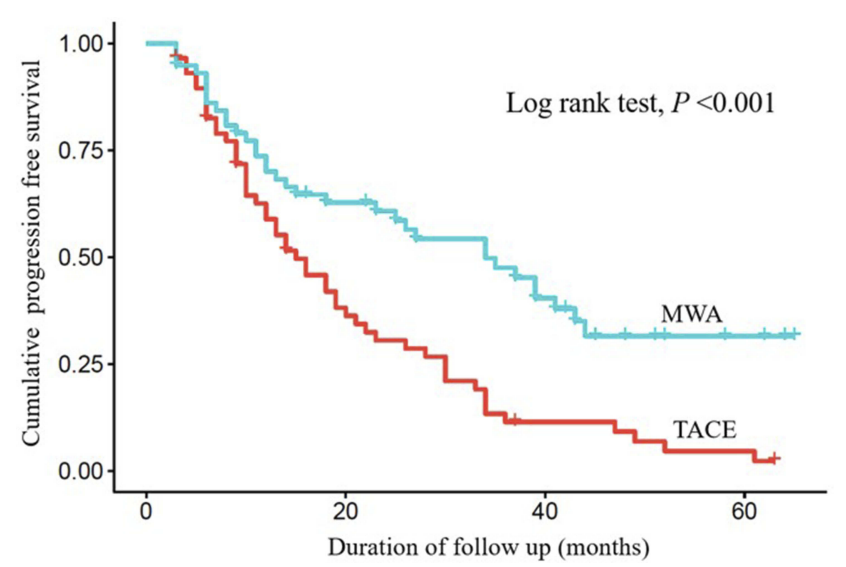

Figure 4 Comparison of the progression-free survival of HCC patients between the MWA group and the TACE group after PSM. Significantly better PFS was found for $\mathrm{HCC}$ patients undergoing MWA.

size. Adequate embolization is relatively easy for lesions with single feeding artery, but more difficult for tumors with complex feeders. ${ }^{27,28}$ However, MWA, due to a lower susceptibility to heat-sink effects and stronger coagulation ability for vessel,,$^{29,30}$ is seldom influenced by the factors in feeding arterials.

For the $3.1-5 \mathrm{~cm}$ lesions, although our results revealed that MWA was not more effective than TACE in OS, the comparative results were clinically relevant. The comparison in PFS showed that MWA is superior to TACE, which makes it a favorable treatment modality in improving the life quality of patients and saving medical expanse. Besides, it cannot be completely confirmed by this retrospective observational study that MWA was not superior to TACE in OS. Further prospective study with longer follow-up periods will help to get a more powerful conclusion.

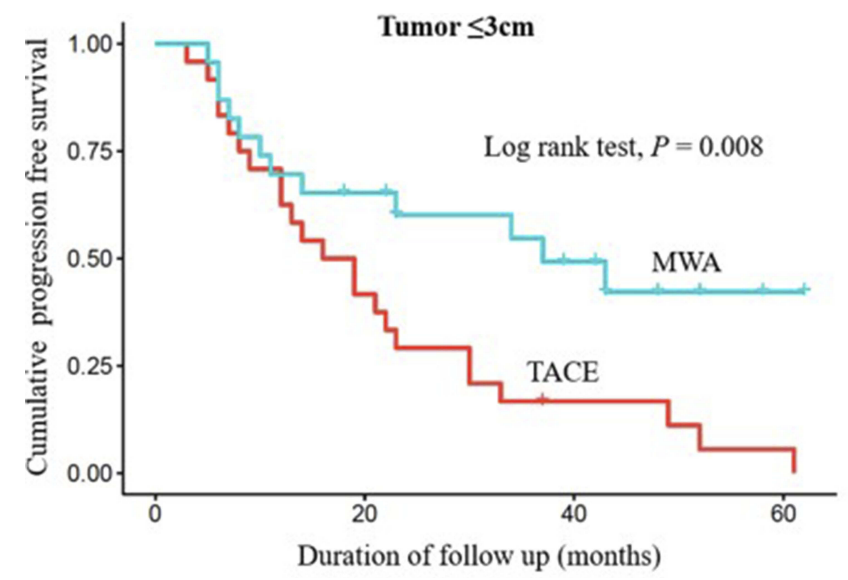

In terms of complications, no significant difference in major complication rate between the MWA group and the TACE group. This was in accordance with the results from other similar comparative studies ${ }^{31}$ and suggested that both MWA and TACE were safe treatment for solitary HCC less than $5 \mathrm{~cm}$.

This study has some limitations. First, inherent selection bias cannot be completely avoided in this cohort study. To reduce the influence of this bias on the comparative outcomes in OS and PFS, propensity score matching analysis was applied. Second, CECT was used to evaluate the response of TACE, which may inevitably overestimate the efficacy of TACE because of the interference from the iodized oil in the judgement of hyper-enhancement of tumor. However, although the $\mathrm{CR}$ rate of TACE was relatively high in this retrospective study, the superiority of MWA remained in the comparison in treatment response. Third, our results may not be representative of the entire world because this study was conducted in single medical center.

In conclusion, results of this study showed that MWA outperformed TACE for solitary HCC smaller than $5 \mathrm{~cm}$ in $\mathrm{OS}$ and PFS, especially for the tumors of $3 \mathrm{~cm}$ or smaller. In clinical practice, for single tumors less than $5 \mathrm{~cm}$, especially those smaller ones $(\leq 3 \mathrm{~cm})$, priority should be given to MWA when making treatment options between MWA and TACE. Besides, when TACE is applied as an initial treatment, more endeavor should be taken to achieve complete response and closed follow-up for disease progression should be underwent.

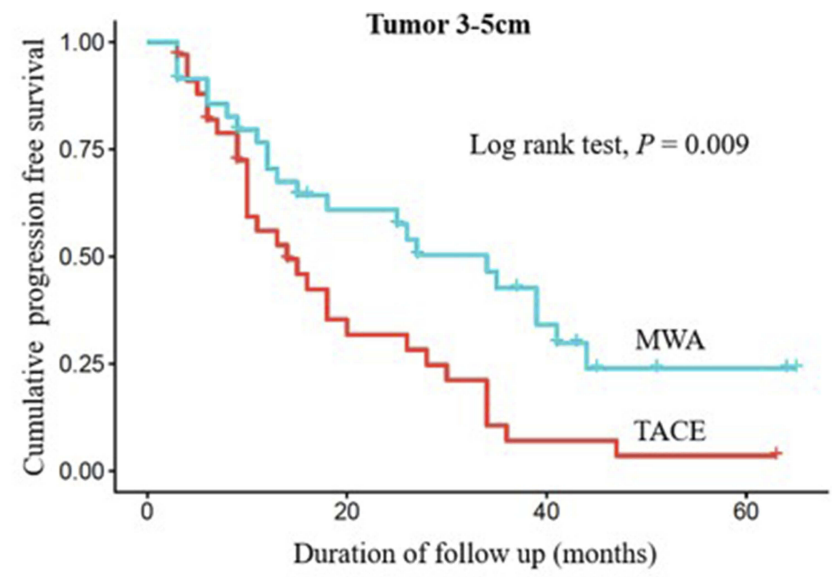

Figure 5 Subgroup analysis of the progression-free survival of HCC patients undergoing MWA or TACE based on the tumor size ( $\leq 3$ or $3.1-5 \mathrm{~cm})$. MWA provides better PFS than TACE for patients in the two subgroups. 


\section{Funding}

This work was supported by grants 2017YFC0112000 and 2018ZX10723-204 from the National Key R\&D Program of Ministry of Science and Technology of China, grants $81627803,81622024,81971625$ and 91859201 from the National Scientific Foundation Committee of China and, grant JQ18021 from the National Scientific Foundation Committee of Beijing and Fostering Funds of Chinese PLA General Hospital for National Distinguished Young Scholar Science Fund.

\section{Disclosure}

No potential conflict of interest was reported by the authors.

\section{References}

1. Torre LA, Bray F, Siegel RL, et al. Global cancer statistics, 2012. $C A$ Cancer J Clin. 2015;65:87-108. doi:10.3322/caac.21262

2. Heimbach JK, Kulik LM, Finn RS, et al. AASLD guidelines for the treatment of hepatocellular carcinoma. Hepatology. 2018;67:35 8-380. doi:10.1002/hep.29086

3. European Association for the Study of the Liver. EASL clinical practice guidelines: management of hepatocellular carcinoma. J Hepatol. 2018;69:182-236. doi:10.1016/j.jhep.2018.03.019

4. Benson AB, D'Angelica MI, Abbott DE, et al. NCCN guidelines insights: hepatobiliary cancers, version 1. JNCCN. 2017;15:563-573.

5. Lucchina N, Tsetis D, Ierardi AM, et al. Current role of microwave ablation in the treatment of small hepatocellular carcinomas. Ann Gastroenterol. 2016;29:460-465.

6. Violi NV, Duran R, Guiu B, et al. Efficacy of microwave ablation versus radiofrequency ablation for the treatment of hepatocellular carcinoma in patients with chronic liver disease: a randomized controlled phase 2 trial. Lancet Gastroenterol Hepatol. 2018;3:317-325. doi:10.1016/S2468-1253(18)30029-3

7. Donadon M, Solbiati L, Dawson L, et al. Hepatocellular carcinoma: the role of interventional oncology. Liver Cancer. 2016;6:34-43. doi: $10.1159 / 000449346$

8. Alejandro F, María R, Jordi B. Hepatocellular carcinoma. Lancet. 2018;391:1301-1314. doi:10.1016/S0140-6736(18)30010-2

9. Pomfret EA, Washburn K, Wald C, et al. Report of a national conference on liver allocation in patients with hepatocellular carcinoma in the United States. Liver Transpl. 2010;16:262-278. doi:10.1002/lt.v16:3

10. Park JW, Chen M, Colombo M, et al. Global patterns of hepatocellular carcinoma management from diagnosis to death: the BRIDGE Study. Liver Int. 2015;35:2155-2166. doi:10.1111/liv.12818

11. Brown KT, Do RK, Gonen M, et al. Randomized trial of hepatic artery embolization for hepatocellular carcinoma using doxorubicin-eluting microspheres compared with embolization with microspheres alone. J Clin Oncol. 2016;34:2046-2053. doi:10.1200/ JCO.2015.64.0821

12. Terzi E, Golfieri R, Piscaglia F, et al. Response rate and clinical outcome of HCC after first and repeated cTACE performed "on demand". J Hepatol. 2012;57:1258-1267. doi:10.1016/j.jhep.2012.07.025

13. Yang HJ, Lee JH, Lee DH, et al. Small single-nodule hepatocellular carcinoma: comparison of transarterial chemoembolization, radiofrequency ablation, and hepatic resection by using inverse probability weighting. Radiology. 2014;271:909-918. doi:10.1148/radiol.13131760

14. Lee SH, Jin YJ, Lee JW. Survival benefit of radiofrequency ablation for solitary $(3-5 \mathrm{~cm})$ hepatocellular carcinoma. Medicine. 2017;96:44.
15. Kim JW, Kim JH, Sung KB, et al. Transarterial chemoembolization vs. radiofrequency ablation for the treatment of single hepatocellular carcinoma $2 \mathrm{~cm}$ or smaller. Am J Gastroenterol. 2014;109:1234-1240. doi:10.1038/ajg.2014.152

16. Hsu CY, Huang YH, Chiou YY, et al. Comparison of radiofrequency ablation and transarterial chemoembolization for hepatocellular carcinoma within the Milan criteria: a propensity score analysis. Liver Transpl. 2011;17:556-566. doi:10.1002/1t.22273

17. Chen RX, Gan YH, Ge NL, et al. Comparison of transarterial chemoembolization with radiofrequency ablation for unresectable Barcelona Clinic Liver Cancer stage 0/A hepatocellular carcinoma: a propensity score matching. $J$ Gastroenterol Hepatol. 2016;31:442-449. doi:10.1111/jgh.13077

18. Liu PH, Lee YH, Hsu CY, et al. Survival advantage of radiofrequency ablation over transarterial chemoembolization for patients with hepatocellular carcinoma and good performance status within the Milan criteria. Ann Surg Oncol. 2014;21:3835-3843. doi:10.1245/s10434-014-3831-2

19. Lencioni R, Llovet JM. Modified RECIST (mRECIST) assessment for hepatocellular carcinoma. Semin Liver Dis. 2010;30:52-60. doi:10. 1055/s-0030-1247132

20. Kamath PS, Wiesner RH, Malinchoc M, et al. A model to predict survival in patients with end-stage liver disease. Hepatology. 2001;33:464-470. doi:10.1053/jhep.2001.22172

21. Ahmed M, Solbiati L, Brace CL. Image-guided tumor ablation: standardization of terminology and reporting criteria - a 10-year update. Radiology. 2014;273:241-260. doi:10.1148/radiol.14132958

22. Sacks D, McClenny TE, Cardella JF, Lewis CA. Society of interventional radiology clinical practice guidelines. $J$ Vasc Interv Radiol. 2003;14:S199-S202. doi:10.1097/01.RVI.0000094584.83406.3e

23. Zhang MK, Ma HM, Zhang J, et al. Comparison of microwave ablation and hepatic resection for hepatocellular carcinoma: a meta-analysis. Onco Targets Ther. 2017;10:4829-4839. doi:10.2147/ OTT

24. Facciorusso A, Di Maso M, Muscatiello N. Microwave ablation versus radiofrequency ablation for the treatment of hepatocellular carcinoma: a systematic review and meta-analysis. Int $J$ Hyperthermia. 2016;32:339-344. doi:10.3109/02656736.2015.1127434

25. Chen QF, Jia ZY, Yang ZQ, Fan WL, Shi HB. Transarterial chemoembolization monotherapy versus combined transarterial chemoembolization-microwave ablation therapy for hepatocellular carcinoma tumors $\leq 5 \mathrm{~cm}$ : a propensity analysis at a single center. Cardiovasc Intervent Radiol. 2017;40:1748-1755. doi:10.1007/ s00270-017-1736-8

26. European Association for the Study of the Liver. EASL-EORTC clinical practice guidelines: management of hepatocellular carcinoma. J Hepatol. 2012;56:908-943. doi:10.1016/j.jhep.2011.12. 001

27. Bolondi L, Burroughs A, Dufour JF, et al. Heterogeneity of patients with intermediate (BCLC B) hepatocellular carcinoma: proposal for a subclassification to facilitate treatment decisions. Semin Liver Dis. 2012;32:348-359.

28. Terzi E, Piscaglia F, Forlani L, et al. TACE performed in patients with a single nodule of hepatocellular carcinoma. BMC Cancer. 2014;14:601. doi:10.1186/1471-2407-14-601

29. Wright AS, Lee FT, Mahvi DM. Hepatic microwave ablation with multiple antennae results in synergistically larger zones of coagulation necrosis. Ann Surg Oncol. 2003;10:275-283. doi:10.1245/ ASO.2003.03.045

30. Chu KF, Dupuy DE. Thermal ablation of tumors: biological mechanisms and advances in therapy. Nat Rev Cancer. 2014;14:199-208. doi: $10.1038 / \mathrm{nrc} 3672$

31. Sato M, Tateishi R, Yasunaga H, et al. Mortality and morbidity of hepatectomy, radiofrequency ablation, and embolization for hepatocellular carcinoma: a national survey of 54,145 patients. $J$ Gastroenterol. 2012;47:1125-1133. doi:10.1007/s00535-012-05 69-0 


\section{Publish your work in this journal}

Cancer Management and Research is an international, peer-reviewed open access journal focusing on cancer research and the optimal use of preventative and integrated treatment interventions to achieve improved outcomes, enhanced survival and quality of life for the cancer patient.
The manuscript management system is completely online and includes a very quick and fair peer-review system, which is all easy to use. Visit http://www.dovepress.com/testimonials.php to read real quotes from published authors.

Submit your manuscript here: https://www.dovepress.com/cancer-management-and-research-journal 